\title{
Focal Takotsubo Cardiomyopathy With High-Dose Interleukin-2 Therapy for Malignant Melanoma
}

\author{
Senthil Damodaran, MD, PhD; Ewa Mrozek, MD; David Liebner, MD; and Kari Kendra, MD, PhD
}

\begin{abstract}
High-dose interleukin-2 (IL-2) is an available treatment option for patients with metastatic melanoma or renal cell carcinoma, and is associated with sustained complete and partial responses in a subset of patients. IL-2, however, is not devoid of toxicities, most of which involve the cardiovascular system and manifest as hypotension, arrhythmias, and cardiomyopathy. This report describes an unusual presentation of takotsubo cardiomyopathy in a postmenopausal woman receiving high-dose IL-2 for metastatic melanoma. (J Natl Compr Canc Netw 2014;12:1666-1670)
\end{abstract}

\section{NCCN: Continuing Education}

\section{Accreditation Statement}

This activity has been designated to meet the educational needs of physicians and nurses involved in the management of patients with cancer. There is no fee for this article. No commercial support was received for this article. The National Comprehensive Cancer Network (NCCN) is accredited by the ACCME to provide continuing medical education for physicians.

NCCN designates this journal-based CME activity for a maximum of 1.0 AMA PRA Category 1 Credit(s) ${ }^{\mathrm{TM}}$. Physicians should claim only the credit commensurate with the extent of their participation in the activity.
NCCN is accredited as a provider of continuing nursing education by the American Nurses Credentialing Center's Commission on ACcreditation.

This activity is accredited for 1.0 contact hours. Accreditation as a provider refers to recognition of educational activities only; accredited status does not imply endorsement by NCCN or ANCC of any commercial products discussed/displayed in conjunction with the educational activity. Kristina M. Gregory, RN, MSN, OCN, is our nurse planner for this educational activity.

All clinicians completing this activity will be issued a certificate of participation. To participate in this journal CE activity: 1) review the learning objectives and author disclosures; 2) study the education content; 3 ) take the posttest with a $66 \%$ minimum passing score and complete the evaluation at http://education.nccn.org/ node/58470; and 4) view/print certificate.

Release date: December 12, 2014; Expiration date: December 12, 2015

\section{Learning Objectives}

Upon completion of this activity, participants will be able to:

- List the potential cardiovascular toxicities and adverse events associated with high-dose IL-2 therapy

- Discuss the characteristic clinical presentation of TC

- Evaluate TC as differential diagnosis for patients, particularly postmenopausal women, who present with features suggestive of ischemia while on high-dose IL-2 treatment for metastatic carcinoma
From the Division of Medical Oncology, The Ohio State University Wexner Medical Center, Columbus, Ohio.

Submitted May 20, 2014; accepted for publication August 18, 2014.

The authors have disclosed that they have no financial interests, arrangements, affiliations, or commercial interests with the manufacturers of any products discussed in this article or their competitors.

Correspondence: Senthil Damodaran, MD, PhD, Division of Medical Oncology, The Ohio State University Wexner Medical Center, M365 Starling Loving Hall, 320 West 10th Avenue, Columbus, OH 43210.

E-mail: senthilkumar.damodaran@osumc.edu

\section{EDITOR}

Kerrin M. Green, MA, Assistant Managing Editor, JNCCN—Journal of the National Comprehensive Cancer Network

Ms. Green has disclosed that she has no relevant financial relationships.

\section{CE AUTHORS}

Deborah J. Moonan, RN, BSN, Director, Continuing Education, has disclosed that she has no relevant financial relationships.

Ann Gianola, MA, Manager, Continuing Education Accreditation \& Program Operations, has disclosed that she has no relevant financial relationships.

Kristina M. Gregory, RN, MSN, OCN, Vice President, Clinical Information Operations, has disclosed that she has no relevant financial relationships. Rashmi Kumar, PhD, Senior Manager, Clinical Content, has disclosed that she has no relevant financial relationships.

Maria Ho, PhD, Oncology Scientist/Senior Medical Writer, has disclosed that she has no relevant financial relationships. 
Takotsubo cardiomyopathy (TC), or apical ballooning syndrome, is a transient left ventricular (LV) dysfunction seen in patients with acute emotional or physical stress. ${ }^{1}$ The term takotsubo is derived from the Japanese name for an octopus trap, which is similar in shape to the LV with apical ballooning. Although considered a form of reversible cardiomyopathy by the American College of Cardiology and American Heart Association, TC rarely presents with cardiogenic shock and ventricular arrhythmias that can be fatal.

Although the precise pathophysiology is unclear, TC has been attributed to excess catecholamine-induced microvascular dysfunction leading to myocardial stunning. ${ }^{2,3}$ Approximately one-third of patients present with an antecedent history of severe emotion stress. Common stressors include the loss of loved ones, financial losses, and natural disasters. For these reasons, TC is often referred to as "broken heart syndrome." One-third of all cases occur in the acute medical illness or surgery settings; in other cases, no potential stressors can be identified.

Typically described in postmenopausal women, clinical presentation is often similar to acute coronary syndrome (ACS), with chest pain and concomitant electrocardiogram abnormalities. ${ }^{4}$ Prevalence is estimated at approximately $5 \%$ in women presenting with symptoms suggestive of ACS. ${ }^{5}$ ST-segment elevation in the anterior precordial leads or T-wave inversion with QT prolongation and abnormal Q waves can be seen on electrocardiogram. ${ }^{1,6}$ Mild elevation in cardiac enzymes (troponin, creatine kinase-MB) can also be seen. Echocardiogram shows apical and/or midventricular wall motion abnormalities that do not conform to a single coronary artery distribution. Although cardiac catheterization corroborates apical ballooning and hypokinesis of LV wall, no obstructive coronary artery disease (CAD) can be discerned that accounts for the observed wall motion abnormalities. A variant with isolated midventricular and basal dysfunction with apical sparing has been reported and is termed reverse takotsubo cardiomyopathy. ${ }^{4}$ Mayo Clinic criteria have been proposed for the diagnosis of TC and include (1) transient LV wall motion abnormalities extending beyond a coronary artery distribution and involving the apical and/or midventricular myocardial segments; (2) ST-segment elevation and/or diffuse T-wave inversion or troponin elevation; and (3) absence of obstructive epicardial CAD or angiographic evidence of acute plaque rupture. ${ }^{1}$ Although treatment of TC is largely supportive, negative inotropic agents such as $\beta$-blockers or calcium channel blockers can be beneficial. ${ }^{7}$ This report presents a case of TC in a patient with metastatic melanoma receiving high-dose interleukin-2 (IL-2) therapy.

\section{Case Report}

A 55-year-old Caucasian woman with a history of hypothyroidism presented to her primary care physician in July 2002 for a mole on the anterior right thigh. Biopsy results were consistent with malignant melanoma and she subsequently underwent a wide excision and sentinel lymph node biopsy. The lesion was determined to be a superficial spreading melanoma with dermal invasion, approximately $1.72 \mathrm{~mm}$ in Breslow thickness, Clark level IV, and with a mitotic count of $8 / \mathrm{mm}^{2}$. Excision margins were negative with no evidence of metastatic disease in the sentinel lymph node. The patient was placed on surveillance and was doing well until December 2010 when she experienced a local recurrence involving her right thigh. Biopsy confirmed recurrent melanoma and the tumor tested positive for a BRAF-V600E mutation. Staging studies performed did not reveal any evidence of metastatic disease and she underwent a wide excision in March 2011. Subsequently, the patient opted for adjuvant interferon $\alpha-2 b$ therapy, and received intravenous high-dose interferon, $20 \mathrm{MU} / \mathrm{m}^{2}$ daily 5 times a week for 20 total doses followed by maintenance subcutaneous interferon, $10 \mathrm{MU} / \mathrm{m}^{2} 3$ times a week for a total duration of 1 year. Therapy was well tolerated except for the development of flu-like symptoms, nausea, and fatigue. However, she required a $30 \%$ dose reduction of interferon in December 2011 for increased pain and neutropenia. She completed her last treatment in March 2012. Unfortunately, staging scans a month later showed interval development of multiple new mediastinal and bilateral hilar lymph nodes.

The patient decided to pursue high-dose IL-2 therapy and was scheduled to receive IL-2 at a dose of $600,000 \mathrm{U} / \mathrm{kg}$, every 8 hours for a maximum of 12 doses every 3 weeks. She started course 1 , cycle 1 in May 2012. An echocardiogram performed before administration of high-dose IL-2 showed normal cardiac function with an LV ejection fraction (LVEF) of $65 \%$. She tolerated her first cycle well except for some rig- 
ors. She started cycle 2 (course 1) in June 2012 and received 11 doses, with the last dose held because of hypotension; her medications included levothyroxine and zolpidem. The patient was admitted for course 2, cycle 1 of high-dose IL-2 in July 2012 and felt generally well. She reported no chest pain, shortness of breath, or pedal edema. Laboratory studies performed before course 2 showed a hemoglobin level of $9.7 \mathrm{~g} / \mathrm{dL}$, platelet count of $387 \times 10^{3} / \mathrm{mm}^{3}$, WBC count of $4.1 \times$ $10^{3} / \mathrm{mm}^{3}$ (absolute neutrophil count, 1.3) and normal coagulation profile. The creatinine level was $0.85 \mathrm{mg} /$ $\mathrm{dL}$ with normal electrolyte levels and liver function tests. However, results of an electrocardiogram performed before course 2, cycle 1 of IL-2 showed new T-wave inversions in the anterolateral leads (Figure 1). Therefore, IL-2 administration was stopped and cardiology was consulted. A repeat echocardiogram showed hypokinesis of apical anterior and apical lateral wall segments with an LVEF of 45\% (reduced from $65 \%$ before IL-2) and mild tricuspid regurgitation. Serial cardiac enzymes were normal. A technetium-99m sestamibi (Cardiolite, Lantheus Medical Imaging, North Billerica, MA) stress test showed a small mild distal anterior wall perfusion defect with reversibility. Gated images showed normal chamber size, wall motion, and thickening, with LVEF estimated at $62 \%$. The patient achieved an age-predicted maximum heart rate of $78 \%$ and did not develop any symptoms during the procedure.

Considering these findings, the patient underwent a left heart catheterization with ventriculography. Cardiac catheterization showed normal coronary arteries and LV end-diastolic pressure. Left ventriculogram showed akinesis of the apical inferior wall and hypokinesis of the apical anterior wall. Her abnormal electrocardiogram, echocardiogram, and myocardial perfusion imaging results associated with normal coronary arteries were consistent with focal TC. She was started on metoprolol, and administration of highdose IL-2 was deferred. A repeat echocardiogram 2 months later showed a return of normal LV function with an ejection fraction of $65 \%$. She was placed on observation, and repeat imaging studies have shown stable disease since July 2012.

\section{Discussion}

Although this patient had diffuse T-wave inversions on electrocardiogram, her overall presentation was not classical for TC. She had no symptoms indicative of ACS and had normal cardiac enzymes. However, results of the echocardiogram and coronary angiogram showed apical ballooning and hypokinesis of the LV wall, with normal coronary arteries. The patient experienced complete resolution of LV function with $\beta$-blocker use and cessation of high-dose IL-2, and has experienced stable disease since that time. Because of her history of TC with IL-2, a rechallenge with highdose IL-2 is not planned if disease progression occurs.

High-dose IL-2, although proven to be beneficial in appropriately selected patients experiencing durable complete $(6 \%)$ or partial responses $(10 \%)$, is associated with significant toxicity. ${ }^{8}$ Cardiovascular effects, such as hypotension, tachycardia, and hypoperfusion, are commonly seen because of capillary leak and a decrease in intravascular volume. ${ }^{9}$ Despite the fact that fluid and/or vasopressor support is often required to ensure adequate tissue perfusion, most of the toxicity is not severe and is reversible. Arrhythmias, mostly supraventricular and rarely ventricular, have also been seen with high-dose IL-2. ${ }^{10}$ Additionally, severe myocarditis resulting in fulminant heart failure has been reported. ${ }^{11}$

Compromised LV function, similar to that seen in septic shock, has been reported with high-dose IL-2. ${ }^{12}$ A case report of persistent cardiomyopathy

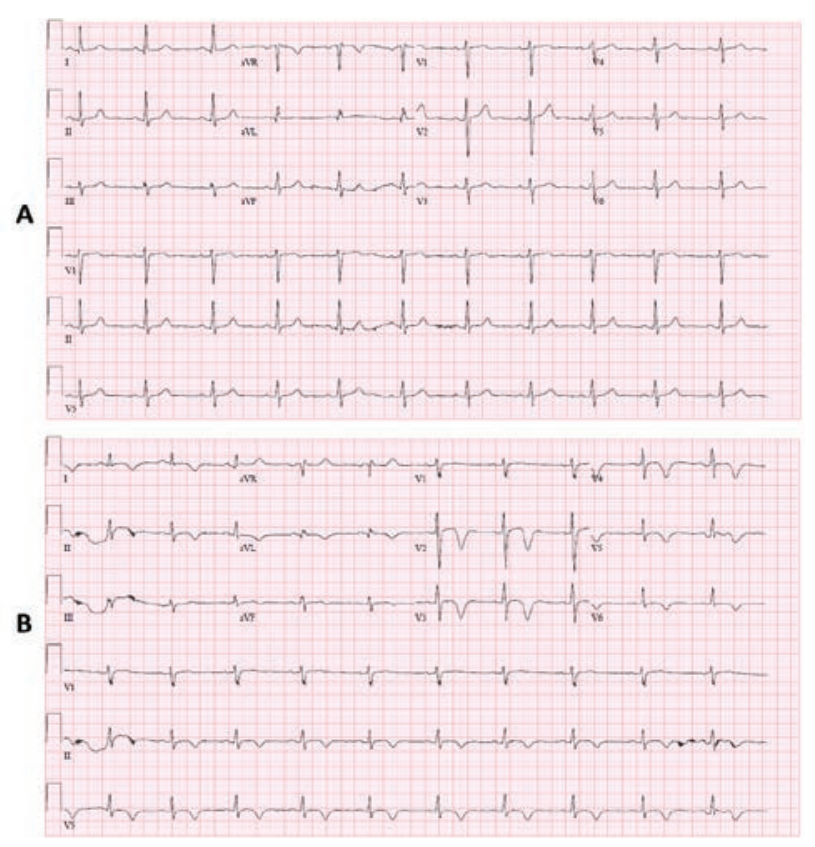

Figure 1 (A) Electrocardiogram findings before high-dose interleukin-2 administration. (B) Electrocardiogram changes after administration of 1 course of high-dose interleukin-2. 
secondary to high-dose IL-2 in a 50-year-old woman has been described. ${ }^{13}$ She had no known cardiac risk factors and received 14 doses of high-dose IL- 2 in the priming course. No improvement in global LV dysfunction was noted, and the patient died on day 59 after administration of IL- 2 because of sudden cardiopulmonary arrest. Although no case of TC with highdose IL-2 has been reported, 2 cases of reversible cardiomyopathy secondary to high-dose IL-2 have been described. ${ }^{14}$ These patients had marked elevation in cardiac enzymes but no ischemic symptoms; however, this was attributed to IL-2-associated myocarditis.

Reversible cardiomyopathy associated with administration of interferon $\alpha-2 b$ has been reported in a 56-year-old man who developed congestive heart failure with interferon $\alpha-2 b$ for malignant melanoma. He developed worsening dyspnea, orthopnea, and fatigue 8 months into therapy. Physical examination and echocardiogram were consistent with severe LV dysfunction, and his symptoms improved with inotropics and cessation of interferon $\alpha-2 b$ therapy. ${ }^{15}$ Coronary angiogram, however, showed nonobstructive stenosis of the proximal right coronary $(60 \%)$ and distal left anterior descending coronary artery (40\%).

Although the present patient was treated with adjuvant interferon $\alpha-2 b$ and high-dose IL-2, her symptoms manifested after starting high-dose IL-2 and she had already completed 1 year of interferon therapy without any cardiac issues.

A few instances of TC have been reported in patients with cancer. Cases of TC, mostly in women, secondary to 5-FU administration for colorectal and gastric carcinomas have been described. ${ }^{16-18}$ Additionally, a 59-year-old man was found to have TC after initially presenting with signs and symptoms indicative of ACS. Interestingly, the development of TC was attributed to a paraneoplastic process secondary to metastatic adenocarcinoma of the lung, and he experienced complete resolution of LV dysfunction with supportive care ${ }^{19}$ Recently, a case was reported of TC that occurred in a patient with metastatic renal cell cancer who was on sunitinib. ${ }^{20}$

\section{Conclusions}

To the authors' knowledge, this is the first reported instance of TC associated with administration of highdose IL-2. A multitude of cardiovascular side effects, including arrhythmias, myocardial infarction, myo- carditis, and cardiomyopathy, can be observed with high-dose IL-2. Patients, including those who are asymptomatic, should be closely monitored for potential side effects, because some are reversible if treated in a timely fashion. TC should be considered as a differential diagnosis for patients, particularly postmenopausal women, who present with features suggestive of ischemia while on high-dose IL-2 treatment.

\section{References}

1. Bybee KA, Prasad A. Stress-related cardiomyopathy syndromes. Circulation 2008;118:397-409.

2. Gianni M, Dentali F, Grandi AM, et al. Apical ballooning syndrome or takotsubo cardiomyopathy: a systematic review. Eur Heart J 2006;27:1523_ 1529.

3. Wickboldt N, Pache JC, Dietrich PY, et al. Takotsubo syndrome secondary to adrenal adenocarcinoma: cortisol as a possible culprit. Am J Respir Crit Care Med 2012;186:1061-1062

4. Richard C. Stress-related cardiomyopathies. Ann Intensive Care 2011;1:39.

5. Anderson JL, Adams CD, Antman EM, et al. ACC/AHA 2007 guidelines for the management of patients with unstable angina/non-ST-elevation myocardial infarction: a report of the American College of Cardiology/ American Heart Association Task Force on Practice Guidelines. J Am Coll Cardiol 2007;50:e1-e157.

6. Ogura R, Hiasa Y, Takahashi T, et al. Specific findings of the standard 12 lead ECG in patients with 'Takotsubo' cardiomyopathy: comparison with the findings of acute anterior myocardial infarction. Circ J 2003;67:687690.

7. Bybee KA, Kara T, Prasad A, et al. Systematic review: transient left ventricular apical ballooning: a syndrome that mimics ST-segment elevation myocardial infarction. Ann Intern Med 2004;141:858-865.

8. Atkins MB, Lotze MT, Dutcher JP, et al. High-dose recombinant interleukin 2 therapy for patients with metastatic melanoma: analysis of 270 patients treated between 1985 and 1993. J Clin Oncol 1999;17:2105-2116.

9. Yeh ET, Tong AT, Lenihan DJ, et al. Cardiovascular complications of cancer therapy: diagnosis, pathogenesis, and management. Circulation 2004;109:3122-3131.

10. White RL Jr, Schwartzentruber DJ, Guleria A, et al. Cardiopulmonary toxicity of treatment with high dose interleukin-2 in 199 consecutive patients with metastatic melanoma or renal cell carcinoma. Cancer 1994;74:3212-3222.

11. Thavendiranathan P, Verhaert D, Kendra KL, Raman SV. Fulminant myocarditis owing to high-dose interleukin-2 therapy for metastatic melanoma. Br J Radiol 2011;84:e99-e102.

12. Ognibene FP, Rosenberg SA, Lotze M, et al. Interleukin-2 administration causes reversible hemodynamic changes and left ventricular dysfunction similar to those seen in septic shock. Chest 1988;94:750-754.

13. Beck AC, Ward JH, Hammond $\mathrm{EH}$, et al. Cardiomyopathy associated with high-dose interleukin-2 therapy. West J Med 1991;155:293-296.

14. Goel M, Flaherty L, Lavine S, Redman BG. Reversible cardiomyopathy after high-dose interleukin-2 therapy. J Immunother (1991) 1992;11:225229.

15. Khakoo AY, Halushka MK, Rame JE, et al. Reversible cardiomyopathy caused by administration of interferon alpha. Nat Clin Pract Cardiovasc Med 2005;2:53-57.

16. Kobayashi N, Hata N, Yokoyama S, et al. A case of Takotsubo cardiomyopathy during 5 -fluorouracil treatment for rectal adenocarcinoma. J Nippon Med Sch 2009;76:27-33.

17. Ozturk MA, Ozveren O, Cinar V, et al. Takotsubo syndrome: an underdiagnosed complication of 5 -fluorouracil mimicking acute myocardial infarction. Blood Coagul Fibrinolysis 2013;24:90-94.

18. Grunwald MR, Howie L, Diaz LA Jr. Takotsubo cardiomyopathy and fluorouracil: case report and review of the literature. J Clin Oncol 2012;30:e11-4.

19. Kepez A, Yesildag O, Erdogan O, Aktas B. Takotsubo cardiomyopathy in a patient with lung adenocarcinoma. Heart Views 2012;13:107-110.

20. Numico G, Sicuro M, Silvestris N, et al. Takotsubo syndrome in a patient treated with sunitinib for renal cancer. J Clin Oncol 2012;30:e218-220. 


\section{Instructions for Completion}

To participate in this journal CE activity: 1) review the learning objectives and author disclosures; 2 ) study the education content; 3 ) take the posttest with a $66 \%$ minimum passing score and complete the evaluation at http://education.nccn.org/ node/58470; and 4) view/print certificate. After reading the article, you should be able to answer the following multiple-

\section{Posttest Questions}

1. True or False: High-dose IL-2 is associated with improved overall survival in patients with metastatic melanoma.

2. Which of the following is indicated for the adjuvant treatment of melanoma?
a. Vemurafenib
b. IL-2
c. Interferon $\alpha-2 b$

choice questions. Credit cannot be obtained for tests completed on paper. You must be a registered user on NCCN.org. If you are not registered on NCCN.org, click on "New Member? Sign up here" link on the left hand side of the Web site to register. Only one answer is correct for each question. Once you successfully answer all posttest questions you will be able to view and/or print your certificate. Software requirements: Internet.

\section{d. All of the above}

3. Which of the following is usually not observed with TC?
a. LV dysfunction
b. Electrocardiogram changes
c. Antecedent history of stress
d. Obstruction of the left anterior de- scending artery

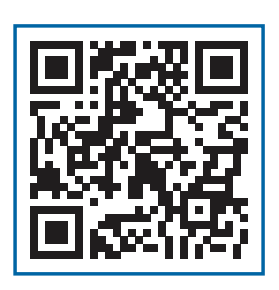

